\title{
INTERCULTURALISMO E COMPETÊNCIA COMUNICATIVA NO ENSINO DE LE
}

Ângela L. F. Ferreira angelaff@infoway.com.br

Márcia J.Dias de Aguiar

amysunbr@yahoo.com.br

\section{RESUMO}

O objetivo deste trabalho é apresentar alguns aspectos referentes à teoria intercultural no que se refere ao desenvolvimento da competência comunicativa dos alunos no contexto de ensino de Língua Estrangeira. Na verdade, busca-se aprimorar o ensino da língua-alvo no sentido de evidenciar os aspectos implícitos de cada cultura através de discussões culturais que possam realçar os contrastes, buscando a superação de mal entendidos e estereótipos negativos. Para isso, utilizamos a teoria de Ratner (1995), Celentin \& Serragioto, Zorzi (1996) como suporte principal para o trabalho.

\section{INTRODUÇÃO}

Ratner (1995), ao postular sobre a influência dos aspectos biológicos nos animais e psicobiológicos nos homens, destaca o papel fundamental da psicologia social na constituição do indivíduo como ser intersubjetivo e cultural. Para o autor, o ser humano se desenvolve socialmente a partir de três aspectos: a consciência, a socialidade, e os instrumentos.

A partir dessa visão de sedimentação e constituição do sujeito, o objetivo deste artigo é detalhar como os três aspectos apresentados por Ratner (1995) contribuem para entender questões de cultura em geral, bem como reportar essas questões para o ensino intercultural de língua estrangeira com ênfase em uma perspectiva de competência comunicativa intercultural participativa.

\section{O SUJEITO SEDIMENTADO PELA MEDIAÇÃO}

Retomando as três categorias de Ratner (1995) que auxiliam a entender como o indivíduo constitui a si mesmo e a sociedade, indicamos a consciência como a representação das atividades mentais do ser humano, o seu pensar e seus processos, sua cognição. O autor (1995:16) realça que a consciência "analisa, sintetiza, delibera, interpreta, planeja, lembra, sente e decide". 
A consciência, de certo modo, está em relação direta com o mundo externo no sentido de que processa mentalmente as percepções e concepções sobre o mundo, influenciando na maneira como iremos compreender e agir nesse mundo.

A respeito da socialidade, verificamos um dos elementos mais importantes na formação social e individual que é a interação, ou seja, a cooperação social que também influencia na maneira como nos relacionamos com o mundo e com os outros. Por outro lado, podemos apontar os instrumentos que, interdependentemente com a consciência e a sociabilidade, auxiliam no processo de construção social.

Segundo o autor (1995:16), "os instrumentos são implementos físicos utilizados para aumentar os poderes naturais do organismo físico". À parte dessa visão materialista da posse de instrumentos, acreditamos que eles podem ser concebidos no nível material bem como no abstrato, pois é óbvio que vivemos e somos auxiliados por instrumentos físicos, porém também o somos intelectualmente ou cognitivamente por instrumentos, muitas vezes não palpáveis, que desempenham um papel importante na nossa formação que seria a linguagem/língua, por exemplo.

Outros instrumentos podem ser vistos tanto no nível material quanto no nível abstrato, por exemplo, no caso dos gêneros de texto. Acreditamos que naqueles gêneros que se apresentam socialmente na forma materializada, como é o caso dos textos representados na escrita, é bem mais visível essa materialização. De outro lado, os gêneros oralizados são de certa forma estáveis, com forma e conteúdo também recorrentes (Bakhtin, 1987). Sendo instituídos socialmente pelo uso sem ensino formal, eles são mais abstratos, não materializados. Algo que merece destaque é que as três espécies de mediação "são interdependentes, inseparáveis, e se reforçam mutuamente." (Ratner, 1995:19).

\section{A RELAÇÃO ENTRE CONSCIÊNCIA, CULTURA E SOCIEDADE}

Nossa principal preocupação nesta subseção é demonstrar a relação intrínseca e efetiva entre a consciência, sociedade e cultura, visto que, segundo Ratner (op.cit), elas são interdependentes. Não seria surpreendente então assinalar que a consciência é estimulada pela sociedade, na medida que os eventos e discursos 
estabelecidos socialmente são processados pela consciência que influencia o comportamento humano.

Nessa perspectiva, as normas e padrões sociais são absorvidos pela consciência que conseqüentemente induz o sujeito a pensar e atuar de determinada forma. Cabe assinalar que na verdade, segundo D'Andrade (1987) e Gee (1999), que trabalham com o conceito de modelo cultural (ou seja, princípios socialmente compartilhados que governam as ações e os pensamentos dos indivíduos), essa consciência no sentido de detalhamento explícito é decididamente imanifesta, pois usamos e seguimos determinados padrões sem necessariamente poder explica-los.

Dessa forma, o que Ratner atribui como consciência não denota exatamente conceitos facilmente explicáveis dos padrões, mas sim algo relacionado ao processo intelectual, cognoscente, simbólico do indivíduo. Naturalmente, então, podemos ver a relação dialógica entre a consciência e a sociedade, sabendo que a consciência também desempenha papel estruturador das configurações sociais.

Assegurando que "a cultura, à parte da referência aos seus artefatos de uma dada comunidade, envolve um conhecimento socialmente adquirido"1 (Alptekin, 1996:53), temos que ela contribui enormemente na produção e desenvolvimento da consciência, sendo auxiliada fundamentalmente pela linguagem. Em outras palavras, conforme Kramsch (1998), a língua/linguagem é responsável por criar cultura e a cultura, por sua vez e dialeticamente, influencia a linguagem.

A linguagem, para Ratner (1995), tem um duplo papel em sua relevância tanto na formação quanto na transmissão da cultura. Além disso, auxilia no desenvolvimento das nossas funções mentais superiores ligadas a consciência. Nesse contexto, a linguagem passa a ser um instrumento crucial na relação entre consciência/pensamento e sociedade/cultura.

De uma forma geral, usamos determinados instrumentos a fim de realizar determinadas funções no nosso contexto, tanto no sentido físico quanto no intelectual. Da mesma maneira que a consciência reflete a sociedade e nela atua, os instrumentos asseguram uma ponte segura para a efetivação dessa relação em equivalência.

\footnotetext{
${ }^{1}$ Tradução da autora: Culture, aside from its reference to the artefacts of a given community, involves socially acquired knowledge.
} 


\section{INTERCULTURALISMO EM SALA DE AULA}

Ao se conceber a teoria previamente exposta e pensar que cultura e sociedade, cultura e contexto estão estritamente associados à linguagem, é de extrema importância no ambiente de ensino de língua estrangeira trazer aspectos culturais a fim de reportar ao aluno uma visão mais completa do que seja o outro/estrangeiro e seu contexto, sua cultura.

Celentin \& Serragioto argumentam que ensinar uma língua estrangeira não é apenas se ater a aspectos lingüísticos, mas sim associa-los a cultura a qual eles representam. Para eles,

uma pessoa que possui um instrumento lingüístico deve também poder contextualizá-lo e conseqüentemente considerar a cultura onde tal instrumento é usado. Isto por que a língua e a cultura são sempre influenciadas reciprocamente. (site, p. 1$)^{2}$

Sendo assim, ao trabalharmos cultura em sala de aula, estaremos proporcionando um ambiente que, até certo ponto, trará o conflito visto que será a cultura do aluno (C1) em contraste com uma segunda (C2). Quando nos referimos a conflito, não estamos conotando algo negativo, mas sim positivo no sentido de que esse choque de pontos de vista, de modos de ver e agir sobre o mundo, se devidamente trabalhado pode proporcionar uma maneira mais respeitosa e acolhedora de ver o outro, bem como de respeitar e aceitar pontos da própria cultura deste último.

$\mathrm{Na}$ tentativa de elaborar ramos de análise que contemplem aspectos culturais, sendo estes relevantes para o aluno de uma LE, torna-se adequado dedicar especial atenção também aos aspectos não verbais sedimentados nessa cultura. Celentin \& Serragioto sustentam que podemos considerar em sala de aula aspectos usuais representativos da linguagem corporal de dado grupo social, prestando atenção a movimentos específicos, gestos, posturas, expressões faciais e assim por diante, que muito significam e podem causar mal entendidos interculturais.

\footnotetext{
${ }^{2}$ Tradução da autora:Una persona Che possiede uno strumento lingüístico deve anche poterlo contestualizzare e quindi considerare la cultura dove tale strumento è usato. Questo perché la lingua e la cultura si sono sempre influenzate vincedevolmente.
} 
Outro foco de atenção seria a língua como objeto, ou seja, segundo os autores, os sinais, desenhos, adornos pessoais, objetos e maneiras de se vestir características de dada cultura. E na representação da língua como ambiente, teríamos as cores, luzes, arquitetura, bem como as noções de espaço e direção.

É interessante destacar que para Ratner (1995) aspectos como a percepção das cores, percepção auditiva, olfativa, espacial, questões de tamanho, as emoções, as necessidades humanas, tudo isso é social e culturalmente constituído, sendo a discussão sobre esses sociotipos em sala de aula algo significativo e esclarecedor.

Levando esses pontos em consideração, Celentin \& Serragioto enfatizam que:

A proposta em classe, entretanto, é importante, pois uma vez estabelecidos quais os valores e quais comportamentos devem ser ensinados, deve-se ver como isso deve ser feito. Uma vez que se entende o vínculo entre doutrina, cultura e língua conjuntamente ao conhecimento das diferenças culturais, distâncias, semelhanças, e como estas influenciam o aprendizado de uma língua, o professor estará inserindo cultura no currículo. (site, p. 4) ${ }^{3}$

Assumindo esta postura, podemos aquilatar a importância de abordar esses aspectos em sala de aula. Para tanto, diversas maneiras estão disponíveis, contudo cabe ao professor, apoiado teoricamente, escolher a opção mais adequada ao contexto. Dentre as possibilidades para o ensino de línguas, podemos usufruir de role-plays, simulações, atividades de leitura crítica que estimulem o pensamento crítico do aluno, atividades comparativas, trabalho com estereótipos, e assim por diante.

\section{A COMPETÊNCIA COMUNICATIVA INTERCULTURAL}

O grande mérito da abordagem comunicativa como instrumento para que o aluno atinja a competência lingüística desejada, segundo Zorzi (1996), é que a atenção, agora, é voltada para o aluno, para as suas necessidades, objetivos, instrumentos necessários para que ele possa interagir lingüisticamente com a cultura do seu interesse. É também fundamental trabalhar com as noções e funções lingüísticas de

\footnotetext{
${ }^{3}$ Tradução da autora: L'approcio in classe, quindi, è importante: uma volta stabiliti quali valori e quali comportamenti devono essere insegnati, si deve vedere come ciò debba essere fatto. Uma volta Che si è capito il legame tra pensiero, cultura e língua, assieme allá conoscenza delle differenze culturali, distanze, somiglianze e come queste influenzino l'apprendimento di uma língua, l'insegnante há inserito la cultura nel curriculum.
} 
que ele deverá fazer uso, assim como as estratégias de comunicação que ele deverá ativar para conseguir uma interação aceitável.

Mais precisamente, a intenção dessa proposta é preparar o aluno de língua estrangeira para que ele chegue o mais perto possível da língua e da cultura do outro, tendo como ideal a proficiência do falante nativo, o que já é extremamente ambicioso para o ambiente de sala de aula. Para Zorzi (1996), o problema é que num curso geralmente não são levadas em consideração as formas possíveis de negociação que o falante nativo pode ou poderia colocar em prática diante do aprendiz, tornando esse processo um caminho de mão única. Ao contrário, para uma interação bem sucedida, é preciso no mínimo dois participantes dispostos a cooperar mutuamente e, especificamente para a participação intercultural, ao menos um de cada cultura. Em suma, é necessária a participação e boa vontade bilateral na construção dessa interação: por mais esforço que um dos dois faça apenas unilateralmente, a experiência didática corre o risco de resultar em fracasso.

Para Zorzi (1996), analisando os encontros interculturais é possível perceber como a interação entre pessoas de diferentes culturas pode ser marcada por momentos de ruptura e/ou conflito (assincronia) como, por exemplo, reações imprevistas, interrupções, suposições, entre outras, que põem periodicamente em xeque o estabelecimento e a manutenção da interação social na qual estão envolvidos, em conseqüência da discrepância entre o 'background' cultural e as regras de comunicação dos envolvidos, discrepância esta que atinge o imaginário de cada participante no que diz respeito à representação que faz de si e do outro na moldura do próprio domínio cultural.

Na concepção de Gee (1999), cada "mundo" (mundo discursivo) está associado a um modelo cultural, que normalmente se traduz e desdobra em teorias, total ou parcialmente inconscientes. Em acordo com essa proposição, Zorzi (1999) afirma que os participantes de encontros interculturais geralmente não têm consciência das experiências socioculturais que estão vivenciando nem das regras de interação que podem ajudar nas suas interpretações, nos momentos de negociação e superação dos conflitos. É comum não estarmos conscientes das convenções e regras mesmo da nossa própria língua e cultura. Então, o ranço que permanece como resultado desses encontros consiste na difusa e constrangedora impressão de uma interação mal-sucedida da qual não sabemos explicar as causas. 
Assim, temos que as competências necessárias para uma comunicação intercultural efetiva são complexas e definitivamente ligadas ao contexto. Zorzi (1996), baseada em Chick (1990), propõe que para que se concretize uma comunicação intercultural adequada é preciso estar consciente das possíveis fontes de assincronia e das prováveis conseqüências negativas que podem resultar dos mal-entendidos. Quando isso acontece, deve-se buscar retrospectivamente no discurso pontos que possam ter causado tais mal entendidos para, assim, pôr em prática estratégias de solução.

Afinal, na concepção de Zorzi (1996, p.4), "a competência comunicativa intercultural consiste em obter uma recíproca adaptação e não somente a adaptação do aluno ao país que o recebe" 4 . Uma pedagogia voltada para o interculturalismo objetiva encontrar estratégias didáticas que sirvam de instrumento para que pessoas de diferentes culturas possam aprender a comunicar-se independentemente das peculiaridades de cada um, sejam elas de comportamento, língua, crenças, etc. Isto implica ver as relações pessoais não apenas como uma oportunidade de aprendizagem lingüística por parte daquele que sabe menos, mas também a oportunidade para que se ampliem as fronteiras culturais e lingüísticas de todos os envolvidos.

\section{A PARTICIPAÇÃO COMO BASE PARA A COMUNICAÇÃO}

Na concepção de Zorzi (1999), a busca por uma comunicação intercultural efetiva envolve todos os participantes no processo, com vistas à superação das dificuldades que se apresentam. Para que isso se efetive em sala de aula, a autora sugere que se deva em primeiro lugar definir a situação, ou seja, tornar explicitas as normas de comportamento verbais e não verbais que se espera do aluno em cada situação, bem como refletir com eles sobre possíveis alternativas aplicáveis a essas mesmas situações, usando as experiências dos próprios alunos e do professor, como forma de conscientizá-los de que um comportamento nem sempre pode ser considerado universal. O aluno deve conhecer também as conseqüências de adotar uma postura que se distancia muito do comportamento do grupo em que ele está inserido ou deseja se inserir. Deve, ainda, ser bem orientado a respeito das normas que regulam a

\footnotetext{
${ }^{4}$ Tradução da autora: La competenza interculturale cosista nel raggiungere um recíproco (e non solo l'adequamento dell'apprendente ai modelli linguistici e culturale Del paese ospitante).
} 
instituição de ensino que contextualiza esse aprendizado: para que elas servem, como funcionam. Tornar claro os hábitos, costumes, crenças e tudo o que envolve a cultura do outro e a em que ele está inserido. Enfim, são estas algumas estratégias ao alcance do professor que podem facilitar sobremaneira os processos de interação social dos alunos, na escola e na sociedade em geral.

A mesma autora defende a tese de que

uma capacidade comunicativa intercultural - comparada com uma competência comunicativa - não possa ser 'ensinada' enquanto se manifesta por meio de comportamentos lingüísticos e não lingüísticos, absolutamente dependentes das infinitas variáveis do conjunto das situações ${ }^{5}$

Para o professor é impossível preparar o aluno para atuar em todas as situações a que ele pode ser exposto, pois elas são dinâmicas e variadas como é a vida cotidiana. Dessa forma, a autora acredita que mesmo que não seja possível ensiná-la, essa capacidade pode ser 'aprendida', desde que se criem situações que favoreçam o estabelecimento de relações positivas com pessoas de culturas diferentes. É necessário que se mude a perspectiva didática: não é só o aluno estrangeiro a ter que adaptar-se ao modelo de comportamento adequado à cultura do país onde ele está vivendo, mas são todos os envolvidos no processo, inclusive professores e alunos do próprio país, que devem entrar num consenso a respeito do comportamento que se espera dele. Isso jamais deve dar a entender que um deva abdicar da sua cultura em favor da do outro, e sim procure buscar formas de negociação nas quais cada um exalte ou atenue aspectos da própria cultura para vir ao encontro do outro.

Na concepção de Morin, devemos

pensar o problema do ensino, considerando por um lado, os efeitos cada vez mais graves da compartimentação dos saberes e da incapacidade de articulálos, uns aos outros; por outro lado, considerando que a aptidão para contextualizar e integrar é uma qualidade fundamental da mente humana, que precisa ser desenvolvida e não atrofiada. (2000, p.16)

\footnotetext{
${ }^{5}$ Tradução da autora: Siamo partiti dall'assunto che uma competenza comunicativa inerculturale- al pari della competenza comunicativa-non passa essere "insegnata", in quanto si manifesta attraverso comportamenti linguistici e non linguistici assolutamente dipendenti dalle infinite variabili Del contesto di situazioni.
} 
Transferindo tal concepção para o ensino de língua estrangeira, podemos relacionála com o mito do ensino de línguas que equaciona forma e uso como objetivos finais, ensino no qual o professor se vê na missão de estimular o aluno a conquistar a proficiência do falante nativo como se somente isso garantisse uma comunicação bem sucedida. Sem dúvida é importante dominar o instrumento da comunicação, oral ou escrita, de uma comunidade-alvo, mas cabe ao professor, além disso e principalmente, evidenciar a importância do contexto e de agir sobre ele. No caso da escola, que está ao seu alcance, trata-se de instigar os alunos nativos a cooperarem com o processo de interação e levar ambos os lados a respeitarem a cultura do outro como possibilidade de crescimento para todos.

\section{CONCLUSÃO}

Reconhecendo a linguagem como instrumento primordial de interação social, capaz de promover o desenvolvimento do homem nos seus aspectos constitutivos que são a consciência e a socialidade, é da maior importância que a escola em geral, e mais especificamente o professor, busque implantar uma didática adequada que efetive esse crescimento.

Para que isso aconteça, na aula de língua estrangeira é relevante evidenciar os aspectos culturais dos "outros" bem como os dos nativos, estimulando a compreensão, a colaboração e o respeito pelas diferenças. Ou seja, promover um contexto onde se construa com esforços bilaterais um processo de negociação que facilite a interação, no qual, além da competência comunicativa, se ampliem também as respectivas fronteiras culturais.

\section{BIBLIOGRAFIA}

ALPTEKIN, Cem. Target-language culture in EFL materials. In: HEDGE, Tricia \& WHITNEY, Norman. Power, Pedagogy \& Practice. Oxford University Press, 1996.

BAKHTIN, Mikhail. Estética da Criação Verbal. São Paulo: Martins Fontes, 1997 CELENTINI, Paola; SERRAGIOTTO, Graziano. II fattore Culturale nell'Insegnamento della Língua. Disponível em: www.irre.toscana.it/italiano_12/materiali/rete.htm 
D'ANDRADE, Roy. A folk model of the mind. In: QUINN, Naomi \& HOLLAND, Dorothy. Cultural Models in Lnaguage and Thought. Cambridge University Press, 1987.

GEE, James. An introduction to Discourse Analysis:Theory and Method. Routledge, 1999.

KRAMSCH, Claire. Language and Culture. Oxford University Press., 1998.

MORIN, Edgar. A Cabeça Bem-feita: repensar a reforma, reformar o pensamento. Rio de Janeiro: Bertrand Brasil, 2000.

RATNER, Carl. A Psicologia Sócio-Histórica de Vygostky: Aplicações Contemporâneas. Porto Alegre: Artes Médicas, 1995.

ZORZI, Daniela. Dalla Competenza Comunicativa allá competenza comunicativa interculturale. In Babylonia 2:1996, 46-52. 